\title{
ADIÇÃO DE NÍQUEL NA SOLUÇÃO NUTRITIVA PARA O CULTIVO DE MUDAS DE UMBUZEIRO(1)
}

\author{
Orlando Sílvio Caires Neves ${ }^{(2)}$, Eric Victor de Oliveira Ferreira ${ }^{(3)}$, Janice Guedes \\ de Carvalho ${ }^{(4)} \&$ Cláudio Roberto Fonsêca Sousa Soares ${ }^{(5)}$
}

\begin{abstract}
RESUMO
Com o objetivo de avaliar a influência da adição de Ni na solução nutritiva de Hoagland \& Arnon sobre o crescimento e a nutrição mineral de mudas de umbuzeiro, realizou-se este trabalho. $O$ delineamento experimental utilizado foi o inteiramente casualizado, com quatro repetições e seis doses de $\mathrm{Ni}(0 ; 0,0005 ; 0,05$; 0,1; 0,5 e 1,0 $\mathrm{mmol} \mathrm{L}^{-1}$ ). Observou-se que o $\mathrm{Ni}$, em pequenas concentrações, estimula o crescimento de mudas de umbuzeiro em solução nutritiva; para o cultivo destas em solução nutritiva de Hoagland \& Arnon, recomenda-se a adição de $0,03 \mathrm{mmol} \mathrm{L}^{-1}$ de Ni.
\end{abstract}

Termos de indexação: umbu, Hoagland, nutrição em Ni e Spondias tuberosa.

\section{SUMMARY: ADDITION OF NICKEL TO NUTRIENT SOLUTION FOR CULTIVATING SPONDIAS TUBEROSE TREE SEEDLINGS}

This study evaluated the influence of $\mathrm{Ni}$ addition to Hoagland - Arnon nutrient solution on the growth and mineral nutrition of Umbu (Spondias tuberose) tree seedlings. The experiment was in a completely randomized design with four replications and six Ni doses $\left(0 ; 0.0005 ; 0.05 ; 0.1 ; 0.5\right.$; and $\left.1.0 \mathrm{mmol} \mathrm{L}^{-1}\right)$. Low Ni concentrations in the nutrient solution stimulated Umbu seedlings growth. The application of $0.03 \mathrm{mmol} \mathrm{L}^{-1}$ of $\mathrm{Ni}$ is recommended for the cultivation of Umbu seedlings in Hoagland - Arnon nutrient solution.

Index terms: Umbu, Hoagland, Ni nutrition, Spondias tuberosa.

\footnotetext{
${ }^{(1)}$ Recebido para publicação em fevereiro de 2006 e aprovado em fevereiro de 2007.

(2) Professor Adjunto da Universidade Federal Rural de Pernambuco - UFRPE. Unidade Acadêmica de Garanhuns. Av. Bom Pastor s/n, Boa Vista, CEP 55296-000 Garanhuns (PE). E-mail: silvio@uag.ufrpe.br

(3) Estudante de Graduação, Bolsista de Iniciação Científica, Universidade Federal de Lavras - UFLA. Caixa Postal 37, CEP 37200 000 Lavras (MG). E-mail: ericsolos@yahoo.com.br

(4) Professora Titular do Departamento de Ciência dos Solos, UFLA. Bolsista CNPq. E-mail: janicegc@ufla.br

(5) Doutorando em Solos e Nutrição de Plantas, DCS/UFLA. Bolsista PRODOC/Capes. E-mal: ???
} 


\section{INTRODUÇÃO}

O umbuzeiro (Spondias tuberosa Arr. Cam.) pertence à família Anacardiaceae. É uma espécie xerófita, caducifólia, originária das zonas menos chuvosas da região Nordeste do Brasil e do norte do Estado de Minas Gerais. Apesar de sua distribuição ser dispersa, o umbuzeiro se consagra como espécie frutífera de grande importância econômica, social e ecológica, cuja cultura representa fonte de renda adicional no período da entressafra, contribuindo com a metade da renda média anual dos agricultores nas áreas de coleta (Araújo et al., 2000).

Presente em grande número de plantas, o Ni foi descrito por Dixon et al. (1975) como componente da enzima urease, despertando na comunidade científica o interesse acerca da sua função em plantas. Desde então, diversas pesquisas relatam respostas à fertilização com $\mathrm{Ni}$, principalmente em soluções nutritivas e meio de cultura (cultura de tecido) (Marschner, 1995; Kabata-Pendias \& Pendias, 2001). Eskew et al. (1983) verificaram que plantas de soja deficientes em $\mathrm{Ni}$ apresentam níveis tóxicos de uréia nas pontas das folhas, ocasionando necrose destas, e isso se deveu à diminuição da atividade da urease. Os mesmos autores afirmaram que a falta de $\mathrm{Ni}$ exerce influência sobre o crescimento e senescência da planta, no metabolismo do $\mathrm{N}$ e na absorção de Fe.

Estudando o efeito do Ni em cevada, Brown et al. (1987) concluíram que ele satisfaz os requerimentos para ser classificado como micronutriente de plantas superiores. Os trabalhos desses autores se concentraram no estudo de três gerações de plantas de cevada, crescidas em solução nutritiva purificada, com ou sem $\mathrm{Ni}$, em que as sementes obtidas na primeira geração foram postas para germinar e crescidas novamente em solução com ou sem Ni. As sementes da terceira geração foram postas para germinar e observou-se que aquelas crescidas nas gerações anteriores, na ausência de $\mathrm{Ni}$, não apresentaram viabilidade, estando o percentual de germinação relacionado com o teor de Ni nos grãos. Assim, os autores observaram que as taxas de germinação dos grãos dependem dos níveis de $\mathrm{Ni}$ fornecidos às plantas-mãe e que as plantas cultivadas sem Ni na solução nutritiva produziram grãos com porcentagem de germinação próxima de zero, ao passo que as plantas cultivadas com $1,0 \mu \mathrm{mol} \mathrm{L}{ }^{-1}$ de Ni na solução nutritiva produziram grãos com porcentagem de germinação superior a $95 \%$.

Considerando-se que o Ni tem função direta na germinação de sementes, que na sua falta esta é comprometida e que ele não pode ser substituído por outro elemento, fez-se necessário o reconhecimento de sua essencialidade. A partir do artigo de Brown et al. (1987), o Ni passou a ser tido como um elemento essencial, e na publicação de Marschner (1995), ele foi incluído na lista dos micronutrientes de plantas. Também, Raven et al. (2001) e Taiz \& Zeiger (2004) apresentam o Ni como micronutriente.
Usualmente, o Ni não entra na composição das soluções nutritivas consideradas completas; entretanto, Taiz \& Zeiger (2004) apresentaram uma variação da solução Hoagland \& Arnon, na qual é fornecido $0,5 \mu \mathrm{mol} \mathrm{L}^{-1}$ de $\mathrm{Ni}\left(0,03 \mathrm{mg} \mathrm{L}^{-1}\right)$, aplicado na forma de $\mathrm{NiSO}_{4} \cdot 6 \mathrm{H}_{2} \mathrm{O}$.

O objetivo deste trabalho foi avaliar a influência da adição de Ni na solução nutritiva Hoagland \& Arnon sobre o crescimento e a nutrição mineral de mudas de umbuzeiro.

\section{MATERIAL E MÉTODOS}

O experimento foi realizado em casa de vegetação no Departamento de Ciência do Solo da Universidade Federal de Lavras, no período de agosto a novembro de 2004. As mudas do umbuzeiro foram oriundas de sementes colhidas de matrizes nativas e germinadas em vermiculita. As sementes passaram pelo processo de quebra de dormência por meio de escarificação mecânica, conforme recomendação de Nascimento et al. (2000).

Após 20 dias da germinação, as mudas atingiram altura média de $9,5 \mathrm{~cm}$ e foram selecionadas quanto à altura e ao vigor, sendo transplantadas para bandejas coletivas de 30 L contendo solução nutritiva Hoagland (Hoagland \& Arnon, 1950), com 25, 50 e 100\% da força iônica, onde permaneceram 15 dias em cada concentração. Transcorrido o período de aclimatação e nova seleção quanto à altura e ao vigor, as mudas foram transplantadas para vasos com capacidade para 3,5 L, contendo uma planta cada.

O delineamento experimental utilizado foi o inteiramente casualizado, com quatro repetições e seis doses de $\mathrm{Ni}\left(0 ; 0,0005 ; 0,05 ; 0,1 ; 0,5\right.$ e $\left.1,0 \mathrm{mmol} \mathrm{L}^{-1}\right)$. Como fonte de Ni utilizou-se o $\mathrm{NiSO}_{4} \cdot 6 \mathrm{H}_{2} \mathrm{O}$. A troca de solução nutritiva foi feita quinzenalmente, usandose água deionizada.

Foram feitas observações visuais dos sintomas de toxidez e, depois de colhido, o material vegetal foi separado em raízes, caule e folhas, lavado em água corrente e destilada, seco em estufa a $70^{\circ} \mathrm{C}$, até peso constante; a matéria seca correspondente a cada uma das partes foi pesada e avaliada.

Após obtenção do extrato nítrico-perclórico, foram determinados os teores de $\mathrm{P}$ por colorimetria; $\mathrm{Ca}, \mathrm{Mg}$, $\mathrm{Cu}, \mathrm{Fe}, \mathrm{Mn}, \mathrm{Zn}$ e Ni, por espectrofotometria de absorção atômica; K, por fotometria de chama; S, por turbidimetria do sulfato de bário; e N total, pelo método semimicro Kjeldahl (Malavolta et al., 1997).

A partir dos teores dos nutrientes no caule e nas folhas e com base na matéria seca destas partes, calculou-se o acúmulo dos elementos na parte aérea das mudas de umbuzeiro.

Os dados obtidos foram submetidos à análise de variância mediante significância do teste $\mathrm{F}$. Para os 
dados que apresentaram diferenças significativas, aplicaram-se análises de regressão, realizadas com o auxílio do programa estatístico SISVAR (Ferreira, 2000).

\section{RESULTADOS E DISCUSSÃO}

\section{Crescimento}

Observou-se que, em todas medidas analisadas (matéria seca das folhas, caule, raízes e total), houve resposta positiva das mudas de umbuzeiro às menores doses de Ni na solução nutritiva, enquanto nas maiores concentrações as mudas acumularam menos matéria seca, indicando efeito tóxico do elemento (Figura 1). Sintomas de toxidez foram observados nas mudas do umbuzeiro, caracterizando-se por clorose generalizada das folhas, seguida de necrose e abscisão. Os sintomas foram observados nas plantas cultivadas em $0,1,0,5$ e 1,0 $\mathrm{mmol} \mathrm{L}^{-1}$ de Ni.

Apesar de os mecanismos de sua fitotoxidez ainda serem pouco estudados, sabe-se que altos teores de $\mathrm{Ni}$ nos tecidos vegetais inibem a fotossíntese e a respiração; os sintomas de efeitos tóxicos relacionamse a lesões nos tecidos, retardamento de crescimento, cloroses e outros sintomas específicos para espécies vegetais (Gupta, 2001).

A matéria seca das folhas das mudas de umbuzeiro atingiu a máxima produção $(24,81 \mathrm{~g} /$ planta) na dose estimada de Ni na solução nutritiva de $0,03 \mathrm{mmol} \mathrm{L}^{-1}$, com acentuado decréscimo a partir desta. Já para a variável matéria seca do caule, a produção máxima $(18,78 \mathrm{~g} / \mathrm{planta})$ foi alcançada na dose estimada de $0,04 \mathrm{mmol} \mathrm{L}^{-1}$.

A exemplo do ocorrido na produção de matéria seca das folhas, as máximas produções da matéria seca de raiz e matéria seca total foram propiciadas pela dose estimada de $0,03 \mathrm{mmol} \mathrm{L}^{-1}$ de Ni. Quanto às raízes, a produção máxima foi de $8,78 \mathrm{~g} / \mathrm{planta}$. Na dose de $0,00 \mathrm{mmol} \mathrm{L}^{-1}$ de $\mathrm{Ni}$, as plantas produziram $28,50 \mathrm{~g} /$ planta de matéria seca total, sendo esta aumentada para $51,76 \mathrm{~g} /$ planta na presença de $0,03 \mathrm{mmol} \mathrm{L}^{-1}$ de Ni, o que representa ganho de $81,5 \%$ de produção. A concentração estimada de Ni na solução nutritiva de $0,03 \mathrm{mmol} \mathrm{L}^{-1}$, que proporcionou
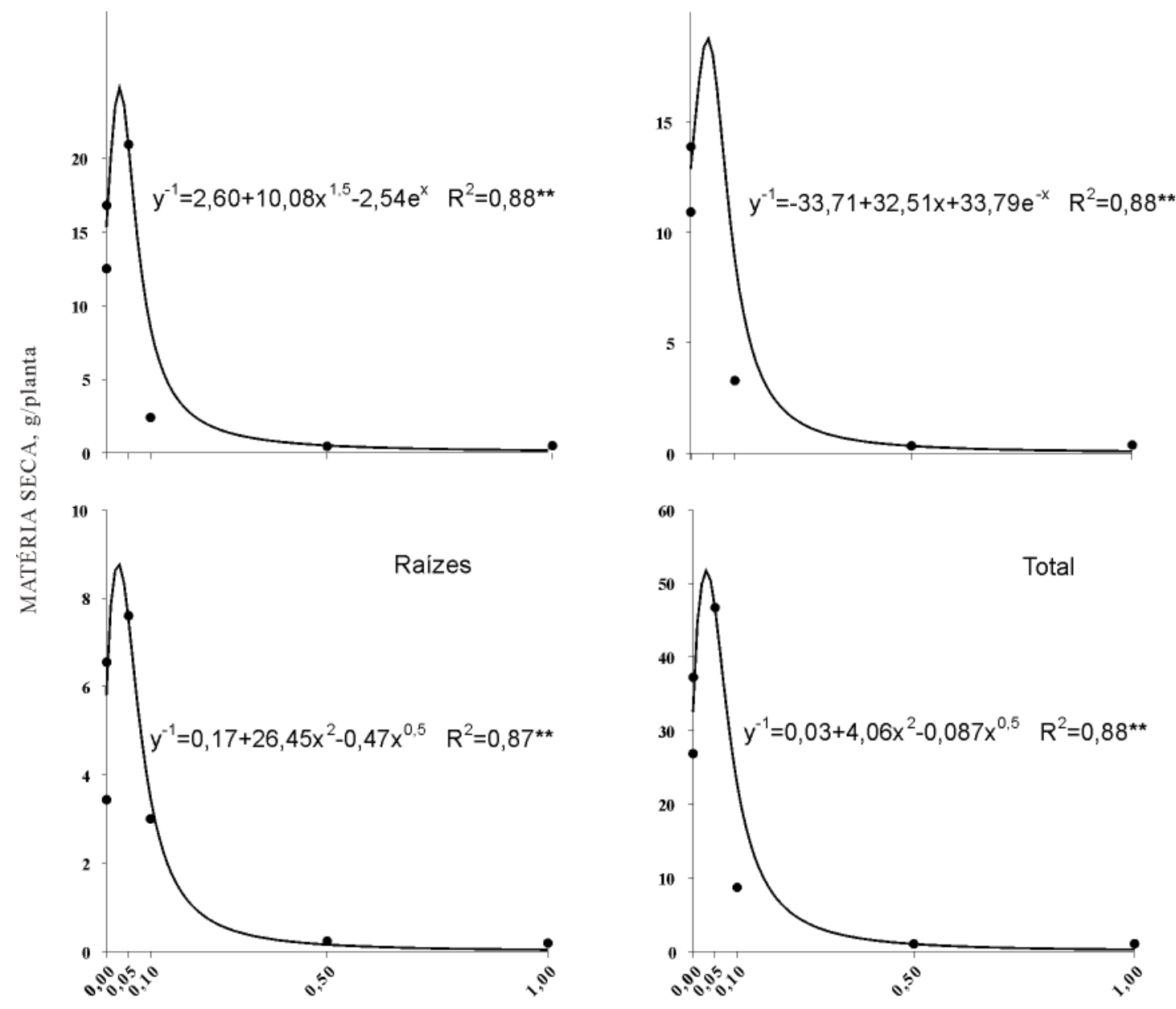

DOSE DE Ni, mmol L $\mathrm{L}^{-1}$

Figura 1. Matéria seca de mudas de umbuzeiro em resposta a doses de níquel na solução nutritiva. (**) significativo a $1 \%$. 
o maior acúmulo de matéria seca pelas mudas do umbuzeiro, é superior à indicada por Taiz \& Zeiger (2004) para as culturas em geral $\left(0,0005 \mathrm{mmol} \mathrm{L}^{-1}\right)$.

\section{Teores de macro e micronutrientes na parte aérea das mudas de umbuzeiro}

Os teores foliares de $\mathrm{N}$ não foram influenciados pelas doses de Ni na solução nutritiva, apresentando média de 27,99 $\mathrm{g} \mathrm{kg}^{-1}$ de $\mathrm{N}$, enquanto os teores dos demais macronutrientes mostram influência delas (Figura 2). Para os teores foliares de $\mathrm{Ca}$, apesar de significativos a $1 \%$, não foi encontrado ajuste para a curva.
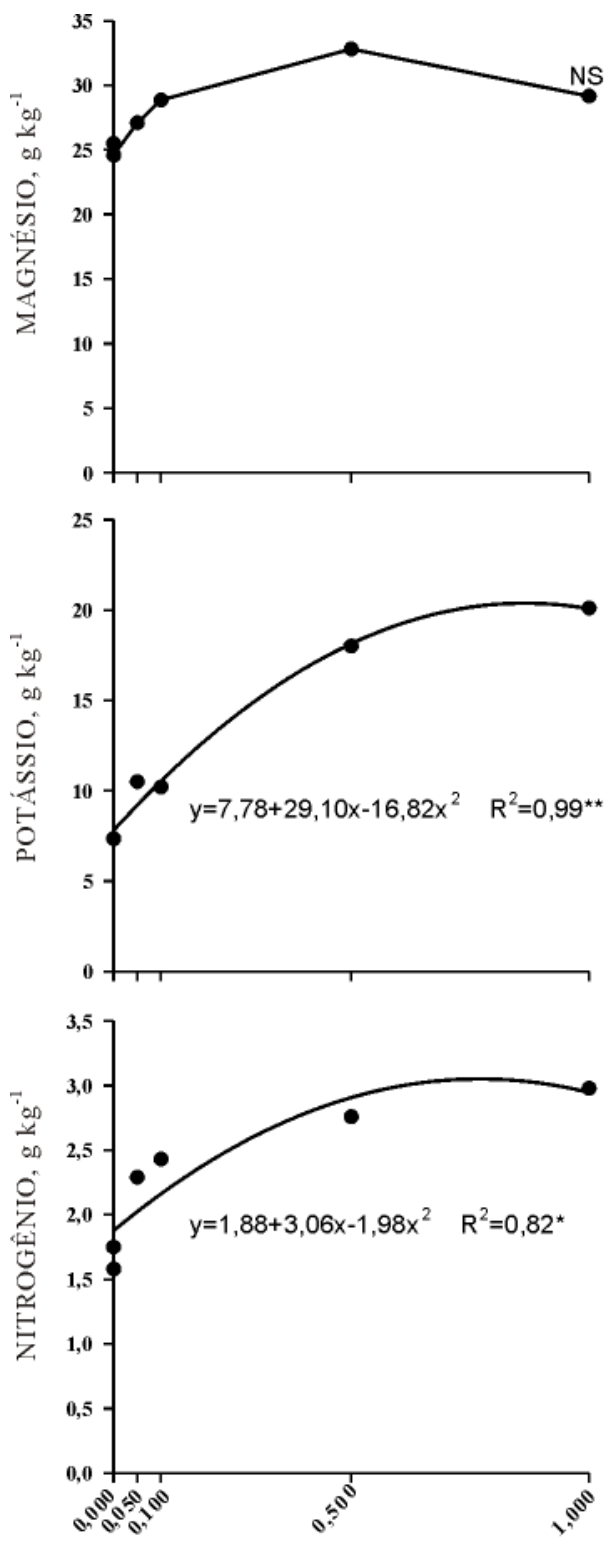

Sabidamente, a influência exercida pelo Ni sobre o metabolismo do $\mathrm{N}$ deve-se ao uso da uréia como adubo ou quando do acúmulo de uréia endógena. O uso de uréia em solução nutritiva não é indicado, pois o principal produto de sua hidrólise, o $\mathrm{NH}_{4}{ }^{+}$, é tóxico às plantas (altos teores) e compete na absorção com outros cátions, ocorrendo ainda redução acentuada do pH da solução. Normalmente, o uso de uréia em solução nutritiva promove redução no crescimento e desenvolvimento das plantas. Entretanto, como demonstrado por Khan et al. (1999) para plantas de espinafre, a adição de $\mathrm{Ni}\left(0,05 \mathrm{mg} \mathrm{L}^{-1}\right)$ em solução juntamente com uréia favorece o crescimento das plantas.
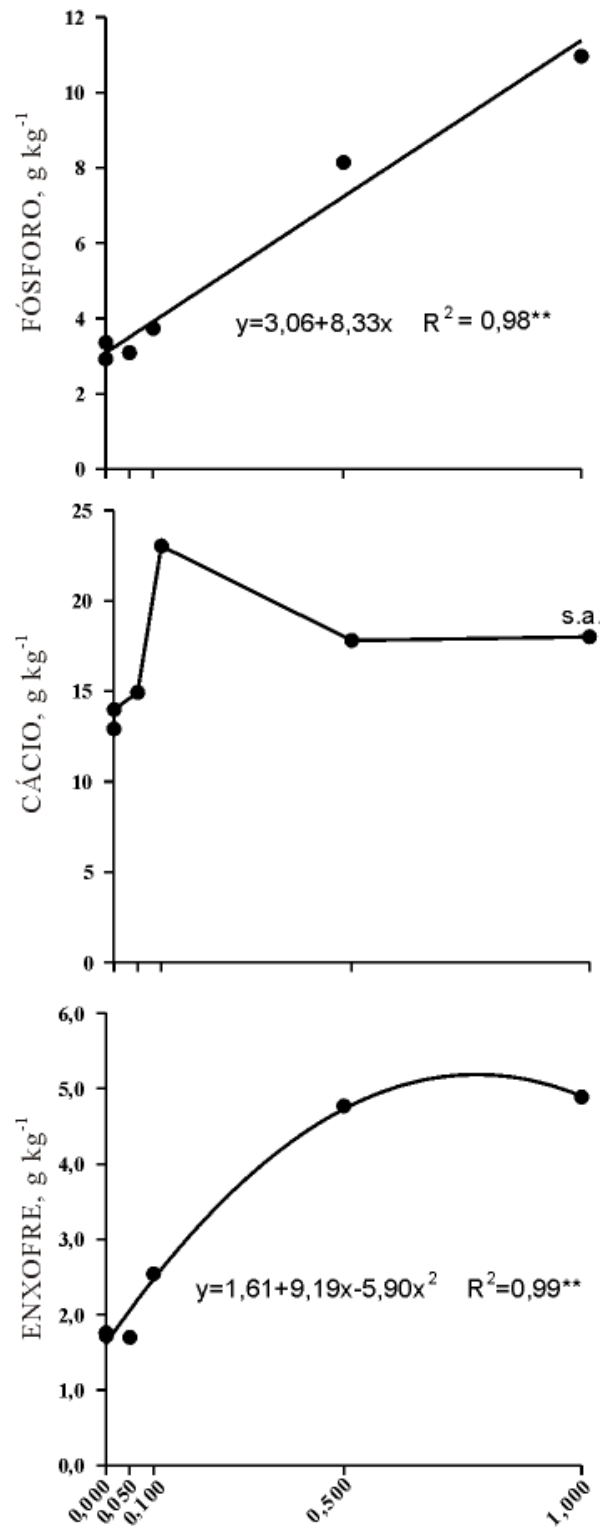

DOSE DE Ni, mmol L ${ }^{-1}$

Figura 2. Teores foliares de macronutrientes em mudas de umbuzeiro em função de doses de níquel na solução nutritiva. (*) e (**), significativos a $5 \%$ e $1 \%$, respectivamente; (s.a.) sem ajuste; (NS) nãosignificativo. 
Os teores foliares de $\mathrm{P}$ em umbuzeiros responderam positiva e linearmente às doses de $\mathrm{Ni}$ na solução nutritiva. Resultado semelhante foi verificado por Paiva et al. (2003), os quais, ao avaliarem a influência de doses de Ni sobre o teor de $\mathrm{P}$ em mudas de ipê-roxo, verificaram que houve aumento nos teores deste elemento, independentemente da parte da planta analisada. No estudo realizado pelos autores citados anteriormente foram utilizadas concentrações de $\mathrm{Ni}$ mais elevadas, sendo o aumento dos teores de P, provavelmente, um efeito de concentração.

Para o K, Mg e S, os seus teores foliares foram aumentados até as doses estimadas de 0,865, 0,777 e $0,778 \mathrm{mmol} \mathrm{L}^{-1} \mathrm{de} \mathrm{Ni}$, respectivamente, apresentando redução a partir destas. O teor máximo de $\mathrm{K}$ foi de $27,99 \mathrm{~g} \mathrm{~kg}^{-1}$; o de $\mathrm{Mg}$, de 3,06 $\mathrm{g} \mathrm{kg}^{-1}$; e o de $\mathrm{S}$, de $5,19 \mathrm{~g} \mathrm{~kg}^{-1}$.

Ao avaliarem a influência de doses de Ni sobre o teor e conteúdo de nutrientes em mudas de ipê-roxo, Paiva et al. (2003) constataram que houve redução nos teores de $\mathrm{Ke} \mathrm{Mg}$, independentemente da parte da planta analisada. Comportamento semelhante foi observado por Palacios et al. (1998), que verificaram redução nos teores de N, P, K, Ca e Mg nas folhas dos tomateiros de acordo com as doses de Ni aplicadas à solução.

Verificou-se que os teores de Ni nas folhas foram influenciados pelas doses desse elemento, sobretudo até o nível de $0,1 \mathrm{mmol} \mathrm{L}^{-1}$, em que ocorreu aumento nos teores de Ni nas folhas (Figura 3). Apesar da significância, não foi encontrado ajuste adequado para a curva.

Os teores foliares de $\mathrm{Ni}$ nas doses de $0,5 \mathrm{e}$ $1,0 \mathrm{mmol} \mathrm{L}^{-1}$ foram superiores aos verificados nas plantas submetidas às doses de $0,00,0,0005 \mathrm{e}$ $0,05 \mathrm{mmol} \mathrm{L}^{-1} \mathrm{de} \mathrm{Ni}$, porém inferiores aos observados na dose de $0,1 \mathrm{mmol} \mathrm{L}^{-1}\left(342 \mathrm{mg} \mathrm{kg}^{-1}\right)$. Salienta-se que, quando cultivadas na presença das três maiores concentrações de Ni na solução $\left(0,1,0,5\right.$ e 1,0 $\left.\mathrm{mmol} \mathrm{L}^{-1}\right)$, as mudas de umbuzeiro apresentaram sintomas de fitotoxidez, que culminaram na redução/paralisação do crescimento das mudas, clorose, necrose e abscisão foliar, sendo os efeitos mais severos observados nas duas maiores concentrações. Assim, esses dois tratamentos foram colhidos antecipadamente em relação aos demais, para que não houvesse perda de material vegetal. Como as plantas do tratamento $0,1 \mathrm{mmol} \mathrm{L}^{-1} \mathrm{de} \mathrm{Ni}$, apesar de apresentarem sintomas de fitotoxidez como clorose e pontos necróticos nas folhas, só foram colhidas ao final do experimento, elas absorveram mais $\mathrm{Ni}$.

O Ni na solução nutritiva influenciou os teores foliares de $\mathrm{Cu}, \mathrm{Fe}, \mathrm{Mn}$ e Zn. Para os teores de $\mathrm{Mn}$ não foi encontrado ajuste adequado. Os teores de $\mathrm{Zn}$ responderam positiva e linearmente às concentrações de Ni na solução nutritiva, enquanto a resposta para os teores de $\mathrm{Cu}$ e $\mathrm{Fe}$ foi quadrática, com seus teores nas folhas das mudas do umbuzeiro aumentando até as doses de 0,709 e $0,593 \mathrm{mmol} \mathrm{L}^{-1}$ de $\mathrm{Ni}$,

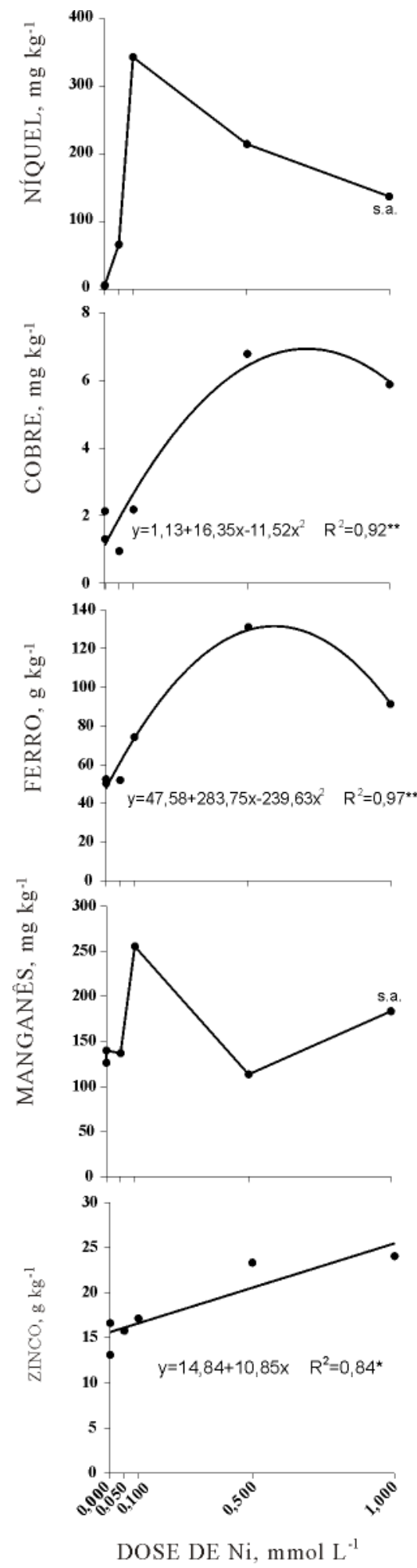

Figura 3. Teores foliares de micronutrientes em mudas de umbuzeiro em função de doses de níquel na solução nutritiva. (*) e (**), significativos a 5 e $1 \%$, respectivamente; (s.a.) sem ajuste.

respectivamente. Nessas doses foram encontrados $6,93 \mathrm{mg} \mathrm{kg}^{-1}$ de Cu e $131,58 \mathrm{mg} \mathrm{kg}^{-1}$ de Fe. Paiva et al. (2003), ao avaliarem a influência de doses de $\mathrm{Ni}$ 
sobre o teor de micronutrientes em mudas de ipê-roxo, também constataram que houve aumento nos teores de $\mathrm{Cu}$ e $\mathrm{Fe}$, independentemente da parte da planta analisada.

Na dose de $0,03 \mathrm{mmol} \mathrm{L}^{-1}$ de $\mathrm{Ni}$, que foi aquela que proporcionou a maior produção de matéria seca pelas mudas de umbuzeiro, os teores estimados de $\mathrm{Cu}$, Fe e $\mathrm{Zn}$ foram de 1,61, 55,87 e 15,15 $\mathrm{mg} \mathrm{kg}^{-1}$, respectivamente. Já para o Ni, os teores aproximados foram de 37,0 e 150,00 $\mathrm{mg} \mathrm{kg}^{-1}$ para o $\mathrm{Mn}$.

Accioly \& Siqueira (2000) relatam duas espécies hiperacumuladoras de Ni: Alyssum bertoloni e Dichapetalum gelonioides; a primeira chega a apresentar teores de $\mathrm{Ni}$ de $13.400 \mathrm{mg} \mathrm{kg}^{-1}$ e a segunda, de $33.000 \mathrm{mg} \mathrm{kg}^{-1}$. Observa-se, pelos resultados do presente trabalho, que o umbuzeiro não apresenta características de planta acumuladora de $\mathrm{Ni}$, porém seus teores foliares $\left(37,0 \mathrm{mg} \mathrm{kg}^{-1}\right)$, verificados na concentração de $0,03 \mathrm{mmol} \mathrm{L}^{-1}$, foram superiores a 10 mg kg-1 - segundo Marschner (1995), o teor acima do qual o Ni pode ocasionar toxidez à maioria das culturas; esse autor destaca que existe alta variabilidade entre as espécies vegetais na capacidade de concentrar Ni nos seus tecidos.

\section{CONCLUSÕES}

1. $\mathrm{O} \mathrm{Ni}$, em pequenas concentrações, estimula o crescimento de mudas de umbuzeiro em solução nutritiva.

2. Para o cultivo de mudas de umbuzeiro em solução nutritiva de Hoagland \& Arnon, recomendase a adição de $0,03 \mathrm{mmol} \mathrm{L}^{-1}$ de $\mathrm{Ni}\left(1,7 \mathrm{mg} \mathrm{L}^{-1}\right)$.

3. Os sintomas de fitotoxidez de Ni observados nas mudas do umbuzeiro foram caracterizados pela redução/paralisação do crescimento e clorose generalizada das folhas, seguida de necrose e abscisão.

\section{LITERATURA CITADA}

ACCIOLY, A.M.A. \& SIQUIERA, J.O. Biorremediação de áreas contaminadas. In: NOVAIS, R.F.; ALVAREZ V., H.V. \& CHEFER, C.E.G.R. Tópicos em ciência do solo. Viçosa, MG, Sociedade Brasileira de Ciência do Solo, 2000. v.1. p.299-352.

ARAÚJO, F.P.; SANTOS, A.C. \& CAVALCANTI, N.B. Cultivo do umbuzeiro. Petrolina, Embrapa, 2000. (Instruções Técnicas da Embrapa Semi-árido, 24)

BROWN, P.H.; WELSH, R.M. \& CARY, E.E. Nickel: A micronutrient essential for higher plants. Plant Physiol., 85:801-803, 1987.
DIXON, N.E.; GAZZOLA, C.; BLAKELEY, R.L. \& ZERNER, R. Jack bean urease. A metalloenzyme. A simple biological role for nickel. J. Am. Chem. Soc., 97:4131-4133, 1975.

ESKEW, D.L.; WELCH, R.M. \& CARY, E.E. Nickel: An essential micronutrient for legumes and possibly all higher plants. Science, 222:621-623, 1983.

FERREIRA, D.F. Análises estatísticas por meio do SISVAR (Sistema para Análise de Variância) para Windows 4. 0. In: REUNIÃO ANUAL DA REGIÃO BRASILEIRA DA SOCIEDADE INTERNACIONAL DE BIOMETRIA, 45., São Carlos, 2000. Anais. São Carlos, Universidade Federal de São Carlos, 2000. p.255-258.

GUPTA, U.C. Micronutrientes e elementos tóxicos em plantas e animais . In: FERREIRA, M.E.; CRUZ, M.C.P.; RAIJ, B. van \& ABREU, C.A., eds. Micronutrientes e elementos tóxicos na agricultura. Jaboticabal, CNPq/FAPESP/ POTAFOS, 2001. p.13-43.

HOAGLAND, D.R. \& ARNON, D.I. The water culture method of growing plants without soil. Berkeley, University. of California, 1950. 32p.

KABATA-PENDIAS, A. \& PENDIAS, H. Trace elements in soils and plants. 3.ed. Boca Raton, CRC Press, 2001. 413p.

KHAN, N.K.; WATANABE, M. \& WATANABE, Y. Effect of different concentrations of urea with or without nickel addition on spinach (Spinacia oleracea L.) growth under hydroponic culture. Soil Sci. Plant Nutr., 45:569-575, 1999.

MALAVOLTA, E.; VITTI, G.C. \& OLIVEIRA, S.A. Avaliação do estado nutricional das plantas: Princípios e aplicações. 2.ed. Piracicaba, POTAFOS, 1997. 319p.

MARSCHNER, H. Mineral nutrition of higher plants. 2.ed. London, Academic Press, 1995. 889p.

NASCIMENTO, C.E.S.; SANTOS, C.A.F. \& OLIVEIRA, V.R. Produção de mudas enxertadas de umbuzeiro (Spondias tuberosa Arruda). Petrolina, Embrapa Semi-Árido, 2000. 13p. (Embrapa. Circular Técnica, 48)

PAIVA, H. N.; CARVALHO, J.G.; SIQUEIRA J.O.; FERNANDES, A.R. \& MIRANDA, J.R.P. Efeito da aplicação de doses crescentes de níquel sobre o teor e o conteúdo de nutrientes em mudas de ipê-roxo (Tabebuia impetiginosa (Mart.) Standley). Sci. For., 63:158-166, 2003.

PALACIOS, G.; GÓMEZ, I.; CARBONELL-BARRACHINA, A.; NAVARRO PEDREÑO, J. \& MATAIX, J. Effect of nickel concentration on tomato plant nutrition and dry matter yield. J. Plant Nutr., 21:2179-2191, 1998.

RAVEN, P.D.; EVERT, R.E. \& EICHHORN, E.E. Biologia vegetal. 6.ed. Rio de Janeiro, Guanabara Koogan, 2001. $906 \mathrm{p}$.

TAIZ, L. \& ZEIGER, E. Fisiologia vegetal. 3.ed. Porto Alegre, Artmed, 2004. 719p. 